\title{
Ideal Timing of Starting Weight-Bearing After Calcaneal Insufficiency Fracture: A Case Report and Review of the Literature
}

\author{
Hitoshi Imamura, ${ }^{1,2,}{ }^{*}$ Takeshi Mochizuki, ${ }^{2}$ Kosei Kawakami, ${ }^{2}$ and Shigeki Momohara ${ }^{1}$ \\ ${ }^{1}$ Department of Orthopedic Surgery, Institute of Rheumatology, Tokyo Women's Medical University, Tokyo, Japan \\ ${ }^{2}$ Department of Orthopedic Surgery, Kamagaya General Hospital, Chiba, Japan \\ "Corresponding author: Hitoshi Imamura, Department of Orthopedic Surgery, Institute of Rheumatology, Tokyo Women's Medical University, Tokyo, Japan. Tel: +81-352691725, \\ Fax: +81-352691726, E-mail: h-imamu@hotmail.co.jp
}

Received 2015 October 21; Revised 2015 December 28; Accepted 2016 January 25.

\begin{abstract}
Introduction: Criteria for starting weight-bearing on the heel with a symptomatic calcaneal insufficiency fracture have not yet been reported.

Case Presentation: We describe a rare case of a 52-year-old woman with a calcaneal insufficiency fracture who sustained a second ipsilateral calcaneal insufficiency fracture within a short time span. The initial fracture was not evident radiographically, but was detected using magnetic resonance imaging (MRI). The patient rejected our advice to avoid weight-bearing on the heel, instead opting to use a silicone heel orthosis. Although there were no abnormal local findings, the stand on heel test was positive at each subsequent visit until 2 months after her first medical examination. At this time, radiographs showed a sclerotic line; however, a second round of MRI showed a new calcaneal insufficiency fracture anterior to the initial calcaneal insufficiency fracture. The patient then agreed to stop weight-bearing on the heel. Three months after the initial visit, radiographs showed two sclerotic lines, and the stand on heel test became negative for the first time; hence, weight-bearing was permitted. There was radiographic evidence of fracture healing and complete resolution of symptoms 4 months after the initial visit.

Conclusions: The callus formation seen on radiographs is helpful in determining when to start weight-bearing; however, fresh insufficiency fractures of the ipsilateral calcaneus may not be detected by radiography. Since local findings such as tenderness, swelling, and heat are subjective, the criteria for starting weight-bearing on the affected heel with an insufficiency fracture should be based on not only radiographs but also objective clinical findings such as the stand on heel test.
\end{abstract}

Keywords: Calcaneus, Insufficiency, Fracture, Timing, Weight-Bearing, Orthosis

\section{Introduction}

Insufficiency fractures are produced by normal or physiological stress applied to bone with deficient elastic resistance (1). Although an insufficiency fracture of the calcaneus is not uncommon among patients with low bone mineral content (2-8), the appropriate time delay before applying a load on the affected heel has not been previously reported.

We describe a rare case of a patient with a calcaneal insufficiency fracture who sustained another ipsilateral calcaneal insufficiency fracture within a short period owing to her failure to protect weight-bearing on the heel. This case gave us insight into the appropriate treatment of a calcaneal insufficiency fracture.

\section{Case Presentation}

The patient was a 52-year-old woman without a history of illness. She presented with pain and swelling of the right ankle and heel; these symptoms had been present for a month and had limited her activities. Although the patient denied any local trauma, she typically stood all day because of her job as a server.

Physical examination revealed tenderness, swelling and warmth of the right ankle and heel, and the stand on heel test (8) was positive. Radiographs of the ankle did not show definite signs of fracture (Figure 1A); however, subsequent magnetic resonance imaging (MRI) of the ankle showed a linear fracture of low signal intensity in the right calcaneus surrounded by bone marrow edema (Figure 1B). The bone mineral density (BMD) of the femoral neck was $0.674 \mathrm{~g} / \mathrm{cm}^{2}$; the T-score was -2.2 standard deviations.

We diagnosed an insufficiency fracture of the calcaneus due to a loss of BMD. The patient was advised to avoid weight-bearing on the heel; however, she adamantly rejected this advice owing to her determination to continue working. Hence, we implemented treatment with a silicone heel cushion orthosis. The patient kept working while using this orthosis, and we closely monitored her condition.

Two months after the first examination, radiographs of

Copyright (C) 2016, Kashan University of Medical Sciences. This is an open-access article distributed under the terms of the Creative Commons Attribution-NonCommercial 4.0 International License (http://creativecommons.org/licenses/by-nc/4.0/) which permits copy and redistribute the material just in noncommercial usages, provided the original work is properly cited. 
the ankle showed a sclerotic line traversing the long axis of the calcaneus (Figure 1C). The tenderness, swelling and warmth of the right ankle and heel had also resolved. However, the stand on heel test was still positive. Therefore, we persuaded the patient to undergo a second round of MRI. The MRI findings of the ankle showed two calcaneal insufficiency fractures (Figure 1D), indicating that another ipsilateral calcaneal insufficiency fracture had developed. Based on this result, the patient accepted our instruction to protect the heel from weight-bearing.

Three months after the first visit, the symptoms had subsided and the stand on heel test finally became negative. Radiographs of the ankle showed two sclerotic fracture lines (Figure 1E). Based on these observations, we gave permission to fully weight-bear on the heel.

There was complete resolution of symptoms 4 months after the initial visit; radiographs of the ankle showed partial displacement of the fractured part of the bone, but healing of the fractures was evident (Figure 1F).

\section{Discussion}

A calcaneal insufficiency fracture is probably more common than is generally acknowledged (9). However, repeated insufficiency fractures in the ipsilateral calcaneus are rare. Calcaneal stress fractures including fatigue fractures and insufficiency fractures are generally accepted to heal with activity restriction, heel-pad inserts, and protected weight-bearing for a short period of time (10). Our therapeutic approach using a heel-pad insert for the initial phase of calcaneal insufficiency fracture treatment resulted in another ipsilateral calcaneal insufficiency fracture within a short time span.

There is a lack of detail in the treatment of calcaneal insufficiency fractures, such as the appropriate time period of non-weight-bearing and the criteria for starting weight-bearing on the heel. Several conservative managements have been previously reported (Table 1$)(2-8,11)$. Most authors selected therapy regimens that limited activity or protected the heel from weight-bearing, although few authors have mentioned the criteria for starting weightbearing.

In this case, the initial therapy using an orthosis was instituted out of regard for the patient's personal reasons. The silicone heel cushion orthosis we used as a heel-pad insert is known to absorb shock $(12,13)$; however, this orthosis was not able to adequately reduce weight-bearing load on the heel, especially as the patient was standing for long periods (12). The occurrence of the second fracture may indicate that the silicone heel cushion orthosis changed the load transfer mechanism; a change in load vector of the calcaneus may mend a rear insufficiency fracture and result in a front insufficiency fracture. Given the assumption that the patient continued applying a load on her affected heel without a heel-pad insert, additional displacement of her posterior part of the calcaneal tuberosity might be shown. Thus, protected weight-bearing appears to be needed to treat symptomatic calcaneal insufficiency fracture.

Criteria for starting weight-bearing on the heel with a symptomatic calcaneal insufficiency fracture have not yet been reported. Table 1 shows that the periods of nonweight-bearing and criteria for starting weight-bearing has varied between patients $(2-8,11)$. This is presumably due to differences in each individual's constitution, periods from pathogenesis to consultation, and treatment environment. Hence, it is inadvisable to assume that there should be a uniform length of non-weight-bearing period for all patients.

We physically examined the patient at every visit, and only the stand on heel test was positive the whole time until 2 months after her first medical examination. At this time, radiographs showed a sclerotic line (Figure 1C); however, MRI showed a new calcaneal insufficiency fracture anterior to the initial calcaneal insufficiency fracture (Figure 1D). This is because MRI is more useful for detecting insufficiency fractures than plain radiography $(4,10)$. Applying a load to the affected heel by referring only to radiographs and local findings such as tenderness, swelling and warmth at this stage may therefore produce an unexpected result. However, it is not common practice to routinely conduct MRI examination due to the associated costs. One month after the patient started non-weight-bearing, the stand on heel test had become negative, and a new sclerotic line had appeared located anterior to the previous sclerotic line (Figure $1 \mathrm{E}$ ).

The stand on heel test excels in screening and detection of calcaneal insufficiency fractures. This test is positive when heel pain is elicited by bearing weight on the heel, and is therefore easy to judge as positive or negative; the reported false-positive rate is $4 \%$ (8). In this case, the patient had continued applying a load to the affected heel using an orthosis even though the stand on heel test was positive; this situation may lead to a prolonged treatment period and partial displacement of the fractured part. Plain radiographs and the stand on heel test are valuable in determining when to start weight-bearing on the calcaneus with an insufficiency fracture, since local findings such as tenderness, bloating and feelings of warmth are subjective.

\subsection{Conclusions}

Criteria for starting weight-bearing on the heel with insufficiency fractures should be mainly based on relatively objective clinical findings such as the stand on heel test. 
Figure 1. A, Radiograph; B, T1-Weighted MRI at the Initial Visit, C, Radiograph; D, T1-Weighted MRI Taken 2 Months After the Patient's Initial Examination; E, Radiograph Taken 3 Months After Initial Examination; F, Radiograph Taken 4 Months After Initial Examination
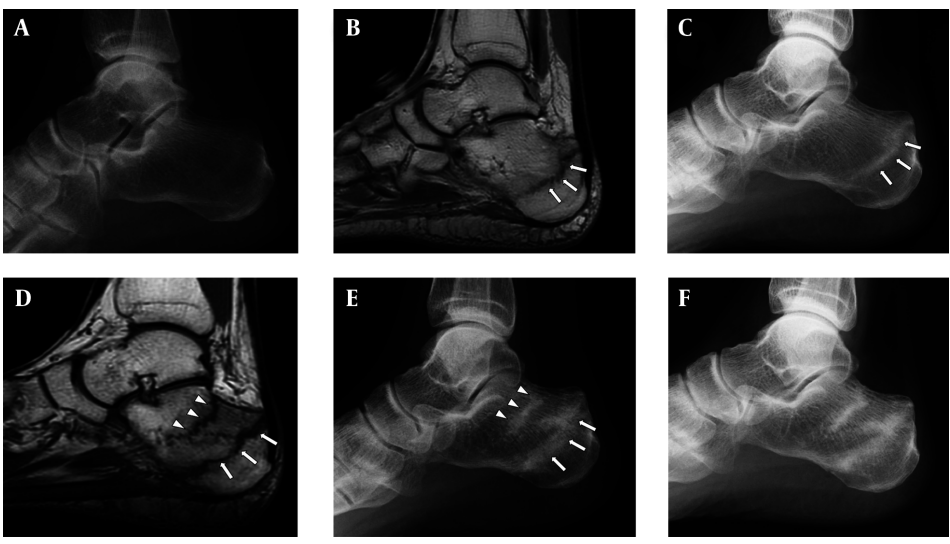

Arrows demonstrate the first insufficiency fracture and arrow heads demonstrate the second insufficiency fracture.

Table 1. Review of the Literature of Conservative Treatment for Calcaneal Insufficiency Fracture Including the Type of Treatment, the Time of Non-Weight-Bearing, and the Criteria for Starting Weight-Bearing

\begin{tabular}{|c|c|c|c|c|c|}
\hline Study Author(s)/Patient Sex & Patient Age, $y$ & Type of Treatment & Length of Non-Weight-Bearing Period & Criteria for Starting Weight-Bearing & Results \\
\hline \multicolumn{6}{|l|}{ Miki et al. (8) } \\
\hline $\mathrm{F}$ & 76 & Limitation of activity & . & . & Healed but partially displaced fracture \\
\hline $\mathrm{F}$ & 77 & Protected weight-bearing & $1 \mathrm{mo}$ & $\begin{array}{l}\text { Improvement in local findings (negative } \\
\text { stand on heel test and squeeze test) }\end{array}$ & Excellent \\
\hline $\mathrm{F}$ & 84 & Limitation of weight-bearing & . & . & Excellent \\
\hline $\mathrm{F}$ & 68 & Protected weight-bearing & $2 \mathrm{w}$ & Improvement of local findings & Excellent \\
\hline $\mathrm{F}$ & 79 & Protect weight-bearing & - & - & Excellent \\
\hline \multicolumn{6}{|l|}{ Alonso-Bartolome et al. (4) } \\
\hline $\mathrm{F}$ & 79 & Limitation of activity & . & . & Excellent \\
\hline $\mathrm{F}$ & 66 & Limitation of activity & - & . & Excellent \\
\hline F & 74 & Limitation of activity & - & - & Excellent \\
\hline $\mathrm{F}$ & 83 & Limitation of activity & . & . & Excellent \\
\hline $\mathrm{F}$ & 69 & Limitation of activity & - & - & Excellent \\
\hline $\mathrm{F}$ & 72 & Limitation of activity & . & . & Excellent \\
\hline \multicolumn{6}{|l|}{ Ha et al. (3) } \\
\hline $\mathrm{F}$ & 72 & Limitation of activity & - & $\cdot$ & Excellent \\
\hline \multicolumn{6}{|l|}{ Arni et al. (7) } \\
\hline $\mathrm{F}$ & 83 & Limitation of activity & - & - & Excellent \\
\hline \multicolumn{6}{|l|}{ Spina et al. (5) } \\
\hline $\mathrm{F}$ & 78 & Protected weight-bearing & $12 \mathrm{w}$ & - & Excellent \\
\hline \multicolumn{6}{|l|}{ Iui (6) } \\
\hline $\mathrm{F}$ & 75 & Operation on other parts & - & & - \\
\hline \multicolumn{6}{|l|}{ Ito et al. (2) } \\
\hline $\mathrm{F}$ & 65 & Protected weight-bearing & $8 \mathrm{w}$ & - & Excellent \\
\hline $\mathrm{F}$ & 78 & Protected weight-bearing & $8 w$ & $\begin{array}{l}\text { Observation of computed tomography } \\
\text { scans }\end{array}$ & Excellent \\
\hline \multicolumn{6}{|l|}{ Charles et al. (11) } \\
\hline $\mathrm{F}$ & 26 & $\begin{array}{l}\text { Weight-bearing as pain allowed using } \\
\text { walking boots }\end{array}$ & . & - & Excellent \\
\hline
\end{tabular}

\section{References}

Pathologic Fractures. JAMA. 1964;187:1001-4. [PubMed: 14102934].

1. Pentecost RL, Murray RA, Brindley HH. Fatigue, Insufficiency, and 
2. Ito K, Hori K, Terashima Y, Sekine M, Kura H. Insufficiency fracture of the body of the calcaneus in elderly patients with osteoporosis: a report of two cases. Clin Orthop Relat Res. 2004(422):190-4. [PubMed: 15187856].

3. Ha YJ, Lee SW, Lee SK, Park YB. Clinical images: osteoporotic calcaneal stress fractures mistaken for aggravation of rheumatoid arthritis. Arthritis Rheum. 2013;65(11):2983. doi: 10.1002/art.38088. [PubMed: 23896818].

4. Alonso-Bartolome P, Blanco R, Canga A, Martinez-Taboada VM. Insufficiency fractures of the calcaneus: a diagnostic pitfall for ankle arthritis. J Rheumatol. 2006;33(6):1140-2. [PubMed: 16755662].

5. Spina A, Clemente A, Vancini C, Fejzo M, Campioni P. Spontaneous talar and calcaneal fracture in rheumatoid arthritis: a case report. J Radiol Case Rep. 2011;5(7):15-23. doi: 10.3941/jrcr.v5i7.735. [PubMed: 22470803].

6. Lui TH. Insufficiency fracture of the body of the calcaneus. Foot (Edinb). 2013;23(2-3):93-5. doi: 10.1016/j.foot.2013.02.002. [PubMed: 23523005].

7. Arni D, Lambert V, Delmi M, Bianchi S. Insufficiency fracture of the calcaneum: Sonographic findings. JClin Ultrasound. 2009;37(7):424-7. doi: 10.1002/jcu.20577. [PubMed: 19353549].
8. Miki T, Miki T, Nishiyama A. Calcaneal stress fracture: an adverse event following total hip and total knee arthroplasty: a report of five cases. J Bone Joint Surg Am. 2014;96(2):eee9. doi: 10.2106/JBJS.L.01472. [PubMed: 24430426].

9. Kannus P, Parkkari J, Niemi S, Palvanen M. Epidemiology of osteoporotic ankle fractures in elderly persons in Finland. Ann Intern Med. 1996;125(12):975-8. [PubMed: 8967708].

10. Gehrmann RM, Renard RL. Current concepts review: Stress fractures of the foot. Foot Ankle Int. 2006;27:750-7.

11. Charles LT, Mehdi AM, Baker D, Edwards MR. Bilateral heel pain in a patient with Diamond-Blackfan anaemia. Foot (Edinb). 2015;25(2):1103. doi: 10.1016/j.foot.2015.03.002. [PubMed: 26004126].

12. Sun PC, Wei HW, Chen $\mathrm{CH}$, Wu CH, Kao HC, Cheng CK. Effects of varying material properties on the load deformation characteristics of heel cushions. Med Eng Phys. 2008;30(6):687-92. doi: 10.1016/j.medengphy.2007.07.010. [PubMed:17888713].

13. Folman Y, Wosk J, Shabat S, Gepstein R. Attenuation of spinal transients at heel strike using viscoelastic heel insoles: an in vivo study. Prev Med. 2004;39(2):351-4. doi: 10.1016/j.ypmed.2004.01.030. [PubMed: 15226045]. 